\title{
A LOGICAL PARADOX.
}

I PREBUYG Mr Lewis Carroll's position to be that the problem raised by him in the last number of Afind presents a conflict between common sense and the rules of logic. It appears to me certain that the rules of logic when properly applied agree with the results of common sense.

The two disputants may ngree in expressing the problem in the following form:-

Principal Antecedent:

Carr is out.

Principal Consequents: If Allen is out, Brown is in;

If Allen is out, Brovon is out.

Uncle Joe uses the general method of the reductio ad absurdum, for he disproves the principal antecedent by maintaining that the consequents to which it leads are incompatible. But in reality the two sub-hypotheticals which form his principal consequents are not incompatible. For in saying that two propositions are incompatible we mean that their combination involves a logical impossibility. Now the combination of these sub-hypotheticals does not involve any imposaibility, but involves merely the denial that Allen can be out. In other words, we combine two hypotheticals, having the same antecedent with contradictory consequents, to prove the falsity of the comm on antecedent. Here we interpret the principle of the reductio ad absurdum in precisely the same way as Uncle Joe, but we apply it to the sub-hypotheticals instead of to the principal hypotheticals. Since, then, the two sub-hypotheticals taken separately would prove "Allen is in," the two principal hypotheticals of which these are the consequents prove "If Carr is out Allen is in."

The larger questions relating to the interpretation of the hypothetical have been implicitly answered in the above solution. Mr Lewis Carroll asks whether a hypothetical whose antecodent is false may be regarded as legitimate. Surely the common example "If $A$ then $B$ but not $B \therefore$ not

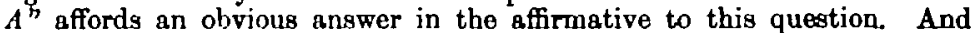
overy reductio ad absurdum argument is a more or lees direct application of the same principle. As regards the hypothetical of the general form "If $\boldsymbol{A}$ then $B$ " we bave interpreted this as the mere denial of the conjunction " $A$ true and $B$ false." The consistent application of this interpretation yields the ahove solution to the whole problem and reduce to equivalence the four proprasitions numerated in Mr Lewis Carroll's note. In other words we must in general interpret a hypothetical in the manner expounded in the article on the Logical Calculus in Mind of January 1892. The solution is, however, independent of any particular views that I have there brought forward, and would be endorsed by all logicians who have applied the rules of logic to complicated problems, though their answers might assume various apparently different forms.

W. E. JoBnson.

\section{A MODIFICATION OF ARISTOTLE'S EXPERIMENT.}

By W. H. R. Rivers.

The generally roceived explanation of the illusion in Aristotle's experiInent is that two parts of the skin are being tunched which in the ordinary position of the fingers have always been touched by two objects. If this is correct, we might expect to find that if two parts of the skin ordiuarily 
touched by one object are simultaneously touched by two objects, there would be the illusive perception of one object, and auch is the case. The illusion cocurs most readily in a person unsware of the nature of the experiment. His eyes should be closed and his fingers aroesed as for Aristotle's experiment. If the outer sides of the croesed fingers (the ulnar side of the inder and the radial side of the middle finger) are simultaneously touched by two rods, such as pencils or penholders, in many cases the obeerver experiences the illusion of hsving one rod between his fingers; "I foel as if you had a single rod going between my two fingers and touching both. The illusion has occurred in more than half of the people with whom I have tried the experiment. It is not so easily obtained as the better known form, but this is the necessary result of several difficulties attending the modified experiment. It is difficult to make the contacts simultaneons, and several observers have noted the successive touches and judged that there were two objeots; if the fingers are not touched on corresponding epots, i.e the index or the ring finger nearer the tip than the middle finger, the illusion may not oocur; again if too much pressure is exerted on the fingers, the obeerver may recognize that such pressure would not arise from one object. Those observers in whom the illusion does not occur, do not usually localize the touches correctly, but suppose that the two contacts are on the opposite sides of one finger.

A very intaresting answer was recoived in one case, vir that three touches were felt, one on each finger, and a third between the fingers, and that the third contact differed from the other two in not being cold (metal rods were being used).

In a note in the first volume of Mind, Croom Robertson pointed out the striking illusion of epatial reference which occur when the crosed fingers are touched one after the other, and was inclined to regard Aristotle's illusion as secondary to this illusory localization in space; "we perceive the contacts as double because we refer them to two distinct parts of вpace." Such an explanation would also apply to this modification, the contacts being perceived as single because we refer them to one part of space. If we cross the inder and middle fingers and hold them vertically, so that the radial side of the middle finger is uppermost, and then with cloeed eyes touch succesively the upper and lower borders, the two touches will be locelized very close to each other, if not actually in the same point in space.

Aristotle's illusion may be regarded as the analogue in the tectile sense of double virion. Here, following an unusual position of the eyes, an object is seen double because it stimulates parts of the rotinse ordinarily stimulated by two objects, and the modification has also its analogue in the visusl sense. When parts of the retinse ondinarily stimulated by one object are stimulated by two objects either by convergence of the eyes to a point nearer than the object or divergence to a point boyond, there is an illusion of one object, the binocular combined image. In the case of vigion however this combined image is accompanied by the two original objects, and it is this which gives especial interest to the observation recorded above in which three touches were felt. Though this phenomenon has only been experienced by one observer, it supports the supposition that the single image which many observers obtain from the two contacta is a combined image analogous to the binocular combined inaga.

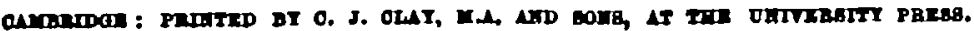

